\title{
Numerical Modelling of Cell Distribution in Blood Flow
}

N. Bessonov ${ }^{1 *}$, E. Babushkina ${ }^{1}$, S.F. Golovashchenko ${ }^{2}$

A. Tosenberger ${ }^{3,4}$, F. Ataullakhanov ${ }^{5,6,7,8}$, M. Panteleev ${ }^{5,6,7,8}$

A. Tokarev ${ }^{5,6}$, V. Volpert ${ }^{3,4,9,10}$

${ }^{1}$ Institute of Problems of Mechanical Engineering, Russian Academy of Sciences 199178 Saint Petersburg, Russia

2 Manufacturing Research Department, Ford Research Laboratory, 481214 Dearborn, USA

${ }^{3}$ Institut Camille Jordan, UMR 5208 CNRS, University Lyon 1, 69622 Villeurbanne, France

${ }^{4}$ INRIA Team Dracula, INRIA Antenne Lyon la Doua, 69603 Villeurbanne, France

${ }^{5}$ National Research Center for Haematology, Ministry of Healthcare of Russian Federation, 125167 Moscow, Russia

${ }^{6}$ Federal Research and Clinical Centre of Paediatric Haematology, Oncology and Immunology

Ministry of Healthcare of Russian Federation, 117198 Moscow, Russia

${ }^{7}$ Faculty of Physics, M. V. Lomonosov Moscow State University, 119991 Moscow, Russia

8 Center for Theoretical Problems of Physicochemical Pharmacology Russian Academy of Sciences, 119991 Moscow, Russia

${ }^{9}$ European Institute of Systems Biology and Medicine, 69007 Lyon, France

${ }^{10}$ Department of Mahematics, Mechanics and Computer Science Southern Federal University, Rostov-on-Don, Russia

\begin{abstract}
Properties of blood cells and their interaction determine their distribution in flow. It is observed experimentally that erythrocytes migrate to the flow axis, platelets to the vessel wall, and leucocytes roll along the vessel wall. In this work, a three-dimensional model based on Dissipative Particle Dynamics method and a new hybrid (discrete-continuous) model for blood cells is used to study the interaction of erythrocytes with platelets and leucocytes in flow. Erythrocytes are modelled as elastic highly deformable membranes, while platelets and leucocytes as elastic membranes with their shape close to a sphere. Separation of erythrocytes and platelets in flow is shown for different values of hematocrit. Erythrocyte and platelet distributions are in a good qualitative agreement with the existing experimental results. Migration of leucocyte to the vessel wall and its rolling along the wall is observed.
\end{abstract}

Keywords and phrases: modelling, erythrocytes, blood flow, dissipative particle dynamics, platelet distribution, leucocyte rolling 
N. Bessonov, E. Babushkina, S.F. Golovashchenko A. Tosenberger, F. Ataullakhanov, M. Panteleev A. Tokarev, V. Volpert Numerical modelling of cell distribution in blood flow

\section{Introduction}

Blood is a complex heterogenous fluid that consists of blood plasma and blood cells. These are red blood cells (RBC) or erythrocytes, white blood cells (WBC) or leucocytes and platelets. Blood cells play a key role in the blood rheology. Various methods are used to model blood flows. They can be split in two main groups, continuous and discrete. Continuous models are based on partial differential equations for Newtonian or non-Newtonian fluids. Blood cells are considered in terms of concentrations with their motion described by diffusion and convection $[5,38,42]$. The disadvantage of this approach is that it does not describe the interaction between individual blood cells in the flow.

Discrete models enable description of individual cells and their interactions. Blood plasma can be described by Navier-Stokes equations or by various particle methods. Erythrocytes are the most interesting and complex blood cells to model because of their deformability. Moreover they constitute $95 \%$ of all cells in blood, and occupy $40 \%$ of the blood volume. There are numerous works devoted to modelling of erythrocytes in blood flow. Specific parachute shape of erythrocytes in the Poiseuille flow was described in $2 \mathrm{D}$ and $3 \mathrm{D}$ RBC membrane models $[10,11,17,27,30,35,41]$. Other experimentally observed effects, $\mathrm{RBC}$ tumbling and tank-threading motion, were described by $3 \mathrm{D}$ simulations in $[6,8,10,11,13,18,35]$.

All these results mainly concern behavior of a single erythrocyte in flow. Much less results were obtained for flows with many erythrocytes. In blood vessels with diameter significantly larger than the diameter of erythrocyte (around $8 \mu \mathrm{m}$ ), it is experimentally observed that erythrocytes concentrate in the bulk of the flow (around the flow axis), while platelets are pushed (migrate) towards the vessel wall. In the case of a vessel wall injury, this mechanism makes the process of clot growth more efficient, as platelets, which are crucial for hemostasis, are located closer to the vessel wall. Several computational studies $[41,45]$ have been done in order to demonstrate mechanical nature of RBC migration to the flow axis. AlMomani et al. [3] used the computational fluid dynamics (CFD) model to perform microscale simulations of platelet-RBC interactions in a shear flow. RBCs were considered as incompressible elliptical particles and platelets as rigid particles of circular shape. It was shown that concentration of platelets increases near the boundary, while erythrocytes are located near the flow axis. Furthermore, it was observed that the migratory effect is absent at very low hematocrit values $\left(H_{t}=5 \%\right)$, but occurs at higher values $\left(H_{t}=10 \%\right)$ and becomes more evident as the hematocrit value increases. Another study [7] was devoted to a two-dimensional numerical investigation of the lateral platelet motion induced by RBCs. There was used a combination of the lattice Boltzmann and Immersed Boundary methods. A deformable elastic RBC's membrane was modelled following Skalak [46] approach, while platelets were modelled as approximately rigid circular objects. It was clearly shown that a near-wall increase in the platelet concentration occurs rapidly (within the first $400 \mathrm{msec}$ ) at the values of hematocrit $20 \%$ and $40 \%$. These works give a correct qualitative description of blood cell distribution. One of their main limitations is that they consider two-dimensional flows instead of the three-dimensional flows.

One more experimentally observed property of blood cells in flow is WBC rolling along the vessel wall. It is involved in immunological response. RBCs and WBCs are cells of approximately equal diameters. The main difference in their mechanical properties is deformability. RBCs are highly deformable, while WBC deformability is very weak. The study in [29] using the lattice Boltzmann approach for 3D simulations showed that the difference in deformability is the reason why RBCs push WBCs to the vessel wall. However, the volume fraction of erythrocytes considered in this work did not correspond to the real blood hematocrit.

This work is devoted to three-dimensional simulations of blood flow with erythrocytes, platelets and leucocytes. We use the Dissipative Particle Dynamics (DPD) method to carry out simulations of blood flow in a cylindrical vessel. DPD is a well developed and widely used approach to mesoscopic description of fluid. RBCs are modelled as elastic highly deformable membranes. In contrast to $[12,14]$, where platelets were modelled as rigid or almost rigid body, we consider them as elastic membranes though its

${ }^{*}$ Corresponding author. E-mail: nickbessonov@yahoo.com 
N. Bessonov, E. Babushkina, S.F. Golovashchenko A. Tosenberger, F. Ataullakhanov, M. Panteleev A. Tokarev, V. Volpert Numerical modelling of cell distribution in blood flow

shape remains close to spherical. WBCs are modelled in the same way as platelets. We study distribution of blood cells and some properties of blood flows.

\section{Numerical model}

\subsection{Spring erythrocyte model}

The RBC model described below is based on properties of its membrane. It consists of a lipid bilayer and spectrin network connected by transmembrane proteins [28]. It is practically incompressible and resistant to the change of surface area and planar shear deformation. The membrane is modelled as a two-dimensional network of particles. They are connected by springs modelled by Hooke's law to form an irregular polyhedron with triangular faces. Forces acting on the membrane particles are similar to [18]. We will refer to this model as Model I and will describe it in this section.

There are four forces acting on particles. One more force related to the DPD method will be introduced below. Let us recall that cell membrane is modelled as a mesh which consists of triangular elements. The first force appears when the sides of the triangles change their length. There are several sides converging to each node and each of these sides contributes to the total force F. Figure 1.2 shows two neighboring nodes and the side connecting them. The force acting on the node $a$ from the node $b$ equals

$$
\mathbf{F}_{s}=k_{s}\left(1-\frac{l}{l_{0}}\right) \boldsymbol{\tau}
$$

where $l$ is the length of the edge between two vertices, $l_{0}$ is the equilibrium length, and $k_{s}$ is the stiffness coefficient, $\boldsymbol{\tau}$ is the unit vector which is codirectional with the vector connecting two neighbouring particles. Furthermore, the forces acting on the nodes $a$ and $b$ have equal absolute values and opposite directions.

Before describing the second force, let us introduce the control volume for each node of the mesh (grey area in Figure 1.1). The outer boundary of the control volume (dotted line) crosses the centers of the sides of the triangle and its center of mass. The control volume of a given node may not belong to the same plane. The mass of the control volume is concentrated at the node. The motion of this node (or particle) is determined by the sum of forces acting on it.

The second force is determined by compression or dilation of the membrane. In order to define it, we introduce in each triangle pressure given by the formula

$$
\mathbf{p}_{2}=k_{a}\left(1-\frac{s}{s_{0}}\right)
$$

where $s$ is the area of triangular element, $s_{0}$ is the equilibrium area, $k_{a}$ is the area expansion modulus. This pressure acts on the outer boundary of the control volumes (Figures 1.1 and 1.3). For example, the force applied to the side de of the boundary of the control volume of the node a equals $\mathbf{F}_{a}=\mathbf{p}_{2} \mathbf{n} l_{d e}$ and $\mathbf{F}_{a}=-\mathbf{F}_{b}$. Similarly other forces at the outer boundaries of the control volumes can be found. These forces are taken into account in the sum of the forces for each node (particle).

Since the out-of-plane bending deformation is present in RBC behavior, bending springs are introduced. The bending moment is considered between each pair of neihgboring triangular elements in the following form:

$$
\mathbf{M}=k_{b} \tan \left(\frac{\theta}{2}\right) l \boldsymbol{\tau}
$$

where $\theta$ is the angle between neighbouring triangular elements (Figure 1.4), $k_{b}$ is the stiffness coefficient, $\tau$ is the unit vectors co-directional with the common edge of two triangles and $l$ is this side's length [18]. 


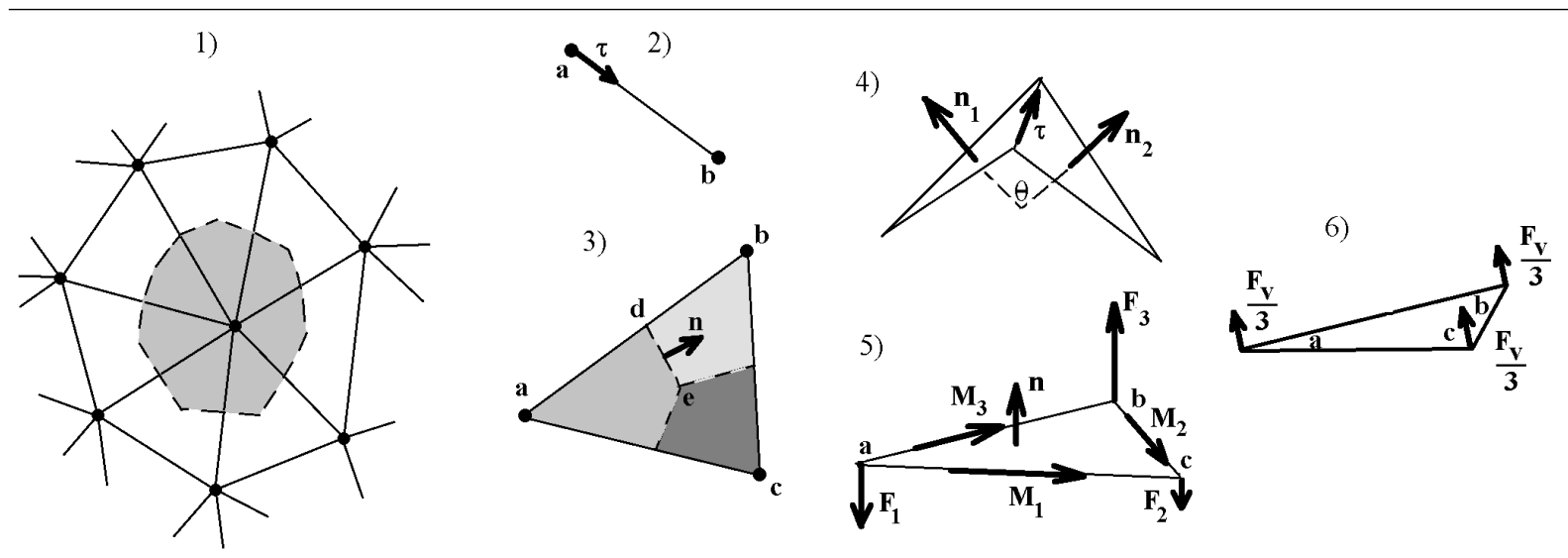

FiguRE 1. Forces acting on nodes of the triangular mesh of cell membrane. 1) Control volume of a node (grey area). 2) The force acting between two nodes depends on the distance between them. It is directed along the side connecting these nodes. 3) The force acting in triangles depends on their area. It is applied to the boundaries of the control volumes. 4) The scheme showing how to determine the direction and the value of momentum between two triangles. 5) Forces at the vertices determined by the action of momentum. 6) Forces at the vertices determined by the change of cell volume.

Each triangle has the total moment from its three sides $\mathbf{M}_{\Sigma}=\mathbf{M}_{1}+\mathbf{M}_{2}+\mathbf{M}_{3}$, (Figure 1.5). In order to find forces corresponding to the moment in a triangle the following system should be solved:

$$
\left\{\begin{array}{l}
\mathbf{F}_{1}+\mathbf{F}_{2}+\mathbf{F}_{3}=0 \\
\mathbf{a}_{1} \times \mathbf{F}_{1}+\mathbf{a}_{2} \times \mathbf{F}_{2}+\mathbf{a}_{3} \times \mathbf{F}_{3}+\mathbf{M}=0
\end{array}\right.
$$

where

$$
\mathbf{a}_{1}=\mathbf{r}_{a}-\mathbf{R}, \mathbf{a}_{2}=\mathbf{r}_{b}-\mathbf{R}, \mathbf{a}_{3}=\mathbf{r}_{c}-\mathbf{R}, \quad \mathbf{R}=\left(\mathbf{r}_{a}+\mathbf{r}_{b}+\mathbf{r}_{c}\right) / 3
$$

$\mathbf{r}_{a}, \mathbf{r}_{b}, \mathbf{r}_{c}$ are radius-vectors of the vertices of the tringle $a b c, \mathbf{F}_{\mathbf{i}}, i=1,2,3$ are the forces that act on the vertices of the triangle (Figure 1.5). All these forces are parallel to the unit vector $\mathbf{n}$. The solution of the system (2.4) has the form:

$$
\left\{\begin{array}{l}
\mathbf{F}_{1}=\frac{\left(\mathbf{a}_{2}-\mathbf{a}_{3}\right) \cdot \mathbf{M}_{\Sigma}}{s} \mathbf{n} \\
\mathbf{F}_{\mathbf{2}}=\frac{\left(\mathbf{a}_{3}-\mathbf{a}_{1}\right) \cdot \mathbf{M}_{\Sigma}}{s} \mathbf{n} \\
\mathbf{F}_{\mathbf{3}}=\frac{\left(\mathbf{a}_{1}-\mathbf{a}_{2}\right) \cdot \mathbf{M}_{\Sigma}}{s} \mathbf{n}
\end{array}\right.
$$

where $\mathbf{n}$ is the normal vector to the surface.

So far, only membrane characteristics have been described, which alone does not ensure the RBC shape. One more force, which depends on the volume of the polyhedron is introduced:

$$
\mathbf{F}_{v}=k_{v}\left(1-\frac{v}{v_{0}}\right) s \mathbf{n}
$$

where $v$ is the polyhedron volume, $v_{0}$ is the equilibrium volume, and $k_{v}$ is the coefficient, $s$ is the area of triangular element and $\mathbf{n}$ is the unit normal vector to this triangle. This force is applied to the vertices of the triangle, $\mathbf{F}_{v} / 3$ to each of them (Figure 1.6). 
N. Bessonov, E. Babushkina, S.F. Golovashchenko A. Tosenberger, F. Ataullakhanov, M. Panteleev A. Tokarev, V. Volpert Numerical modelling of cell distribution in blood flow

\subsection{Modified erythrocyte model}

We suggest a new modified erythrocyte model that combines the continuous description of an elastic body for surface elements and a spring model for the angle between these elements. The membrane is represented as a two-dimensional surface that consists of elastic triangular elements (Figure 2.1).
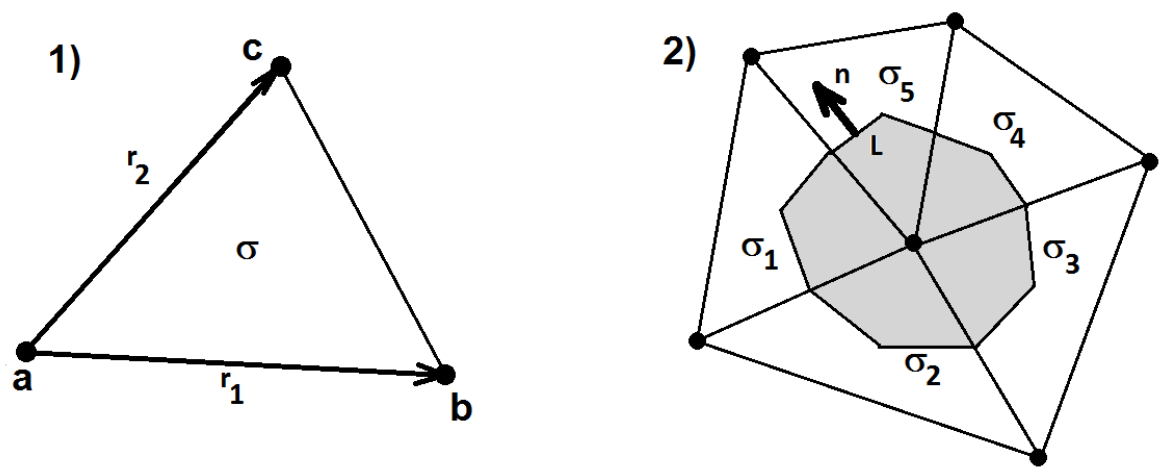

Figure 2. Triangulation of the cell surface for the modified erythrocyte model. 1) Elastic element. 2) Control volume of a particle.

The stress tensor for each triangular element is defined as:

$$
\begin{gathered}
\boldsymbol{\sigma}=-\mathbf{I} p+G(\mathbf{B}-\mathbf{I}), \\
p=K\left(1-\frac{s}{s_{0}}\right),
\end{gathered}
$$

where $p$ is the pressure, $\mathbf{G}$ is the shear coefficient, $K$ is the bulk modulus, $\mathbf{I}$ is a unit tensor, $\mathbf{B}$ is the left Cauchy-Green tensor,

$$
\begin{gathered}
\mathbf{B}=\mathbf{F} \cdot \mathbf{F}^{T}, \\
\mathbf{F}=\frac{d \mathbf{r}}{d \mathbf{R}}=\left|\begin{array}{ll}
\frac{\partial r_{1}}{\partial R_{1}} & \frac{\partial r_{1}}{\partial R_{2}} \\
\frac{\partial r_{2}}{\partial R_{1}} & \frac{\partial r_{2}}{\partial R_{2}}
\end{array}\right|
\end{gathered}
$$

is the deformation gradient, $\mathbf{r}$ is the actual position vector, $\mathbf{R}$ is the initial position vector $(\mathbf{R}=\mathbf{r}$ at $t=0)$.

After formulating the governing equations, let us discuss their approximation [4]. Consider the triangular element (Figure 2). For each triangular element we introduce a local two-dimensional coordinate system. We denote the initial position of its vertices in the local coordinate system at the initial moment of time by the position-vectors $\mathbf{R}_{\mathbf{a}}, \mathbf{R}_{\mathbf{b}}$ and $\mathbf{R}_{\mathbf{c}}$. Due to the deformation process, the vertices move from their initial positions. We denote the actual position of the vertices of the element at time $t$ by $\mathbf{r}_{\mathbf{a}}, \mathbf{r}_{\mathbf{b}}$ and $\mathbf{r}_{\mathbf{c}}$. Let $\mathbf{r}_{\mathbf{i}}$ and $\mathbf{R}_{\mathbf{i}}(i=1,2,3)$ be a set of vectors directed along any two different edges of the triangle, for example:

$$
\mathbf{r}_{1}=\mathbf{r}_{\mathrm{b}}-\mathbf{r}_{\mathrm{a}}, \quad \mathbf{r}_{2}=\mathbf{r}_{\mathbf{c}}-\mathbf{r}_{\mathbf{a}}
$$

and

$$
\mathbf{R}_{1}=\mathbf{R}_{\mathrm{b}}-\mathbf{R}_{\mathrm{a}}, \quad \mathbf{R}_{\mathbf{2}}=\mathbf{R}_{\mathrm{c}}-\mathbf{R}_{\mathrm{a}}
$$


N. Bessonov, E. Babushkina, S.F. Golovashchenko A. Tosenberger, F. Ataullakhanov, M. Panteleev A. Tokarev, V. Volpert Numerical modelling of cell distribution in blood flow

To find the tensor B (see (2.9)) in the element at time $t$ we will use the linear transformation of the element. Then from its initial to actual position

$$
\mathbf{r}=\mathbf{A} \cdot \mathbf{R}+\mathbf{b}
$$

where $\mathbf{A}$, and $\mathbf{b}$ are some parameters of the transformation. For transformation (2.13) the deformation gradient $\mathbf{F}$ is

$$
\mathbf{F}=\frac{\mathbf{d} \mathbf{r}}{\mathbf{d R}}=\frac{\partial \mathbf{r}_{\mathbf{i}}}{\partial \mathbf{R}_{\mathbf{j}}} \mathbf{e}_{\mathbf{i}} \mathbf{e}_{\mathbf{j}}=\frac{\partial\left(\mathbf{A}_{\mathbf{i k}} \mathbf{R}_{\mathbf{k}}+\mathbf{b}_{\mathbf{i}}\right)}{\partial \mathbf{R}_{\mathbf{j}}} \mathbf{e}_{\mathbf{i}} \mathbf{e}_{\mathbf{j}}=\mathbf{A}_{\mathbf{i k}} \delta_{\mathbf{k j}} \mathbf{e}_{\mathbf{i}} \mathbf{e}_{\mathbf{j}}=\mathbf{A}
$$

where $\mathbf{e}_{\mathbf{i}}$ are unit vectors and $\delta_{k j}$ is the Kronecker delta.

As we can see from equation (2.14), in order to determine $\mathbf{F}$ it is necessary to find the tensor $\mathbf{A}$. We can write for the vertices of the element

$$
\left\{\begin{array}{l}
\mathbf{r}_{1}=\mathbf{A} \cdot \mathbf{R}_{1} \\
\mathbf{r}_{2}=\mathbf{A} \cdot \mathbf{R}_{2}
\end{array}\right.
$$

Having solved system (2.15), we can find the tensor A

$$
\mathbf{A}=\mathbf{r}_{\mathbf{i}} \mathbf{R}^{\mathbf{i}}
$$

where vectors $\mathbf{R}_{\mathbf{i}}$ and $\mathbf{R}^{\mathbf{i}}$ are reciprocal.

We can now introduce the approximation for B taking into account (2.14) and (2.16):

$$
\mathbf{B}=\mathbf{F} \cdot \mathbf{F}^{\mathbf{T}}=\mathbf{r}_{\mathbf{s}} \mathbf{R}^{\mathrm{s}} \cdot \mathbf{R}^{\mathrm{k}} \mathbf{r}_{\mathbf{k}}=\mathbf{r}_{\mathbf{s}} \mathbf{e}_{\mathbf{s}} \cdot \mathbf{e}_{\mathbf{m}} \mathbf{R}^{\mathbf{m}} \cdot \mathbf{R}^{\mathrm{k}} \mathbf{e}_{\mathbf{k}} \cdot \mathbf{e}_{\mathbf{j}} \mathbf{r}_{\mathbf{j}}=\mathbf{X}_{\mathbf{r}}^{\mathbf{T}} \cdot \mathbf{G}^{\mathbf{R}} \cdot \mathbf{X}_{\mathbf{r}},
$$

where $\mathbf{G}^{\mathbf{R}}=\mathbf{X}^{\mathbf{R}} \cdot\left(\mathbf{X}^{\mathbf{R}}\right)^{\mathbf{T}}$ is a symmetric tensor with the components $\mathbf{R}^{\mathbf{m}} \cdot \mathbf{R}^{\mathbf{k}}, \mathbf{X}_{\mathbf{r}}=\mathbf{e}_{\mathbf{s}} \mathbf{r}_{\mathbf{s}}$, and $\mathbf{X}^{\mathbf{R}}=\mathbf{R}^{\mathbf{s}} \mathbf{e}_{\mathbf{s}}$.

Having defined $\mathbf{B}$ in an element and defining $p$ from its initial and actual volumes, we calculate the stress tensor in the element from (2.7).

In order to find the elastic force in one vertex we use for each interval $L$ (Figure 2.2) the following equation:

$$
\mathbf{F}_{\mathbf{L}}=\boldsymbol{\sigma} \cdot \mathbf{n} L,
$$

where $\mathbf{n}$ is an outward normal vector to this area. After that we take a sum of forces (2.18) with respect to all intervals in the boundary of the control volume.

Furthermore, instead of the equation (2.7) it is possible to use the formula

$$
\boldsymbol{\sigma}=-\mathbf{I} p+G_{1}(\mathbf{B}-\mathbf{I})+G_{2}\left(\mathbf{B}^{2}-\mathbf{I}\right),
$$

where the stress tensor is the second order function of $\mathbf{B}$. We will refer to the model based on the equation (2.7) as Model II and to the model based on the equation (2.19) as Model III.

In order to describe out-of-plane bending of elements (relative to each other) and conservation of the cell volume, we introduced forces (2.4) and (2.6) for the triangle vertices as it was done in the spring model.

\subsection{Platelet and leucocyte model}

Mechanical properties of platelets and leucocytes differ from properties of erythrocytes. These cells have spherical shape and almost do not deform during motion in blood flow. Platelets are small cells, 2-3 $\mu \mathrm{m}$ in diameter and diameter of leucocytes is 7-8 $\mu \mathrm{m}$. Nevertheless the models that are described in Sections 2.1 and 2.2 allow their description as weakly deformable membranes. Their properties depend on the choice of parameters. We will discuss them in Section 3. Simulations with different cells were carried out using the same model for all cells, however with different values of parameters for cells of different types. 


\subsection{Flow model}

We use the Dissipative Particle Dynamics (DPD) method in the form described in literature $[12,16,20]$. It is a mesoscale method where each DPD particle describes some small volume of a simulated medium rather than an individual molecule. The method is governed by three equations describing the conservative, dissipative and random forces acting between each two particles:

$$
\begin{aligned}
& \mathbf{F}_{i j}^{C}=F_{i j}^{C}\left(r_{i j}\right) \hat{\mathbf{r}}_{i j}, \\
& \mathbf{F}_{i j}^{D}=-\gamma \omega^{D}\left(r_{i j}\right)\left(\mathbf{v}_{i j} \cdot \hat{\mathbf{r}}_{i j}\right) \hat{\mathbf{r}}_{i j}, \\
& \mathbf{F}_{i j}^{R}=\sigma \omega^{R}\left(r_{i j}\right) \frac{\xi_{i j}}{\sqrt{\Delta t}} \hat{\mathbf{r}}_{i j},
\end{aligned}
$$

where $\mathbf{r}_{i}$ is the vector of position of the particle $i, \mathbf{r}_{i j}=\mathbf{r}_{i}-\mathbf{r}_{j}, r_{i j}=\left|\mathbf{r}_{i j}\right|, \hat{\mathbf{r}}_{i j}=\mathbf{r}_{i j} / r_{i j}$, and $\mathbf{v}_{i j}=\mathbf{v}_{i}-\mathbf{v}_{j}$ is the difference between velocities of two particles, $\Delta$ t is the time step in a simulation, $\gamma$ and $\sigma$ are coefficients which determine the strength of the dissipative and the random force respectively, while $\omega^{D}$ and $\omega^{R}$ are weight functions; $\xi_{i j}$ is a normally distributed random variable with zero mean, unit variance, and $\xi_{i j}=\xi_{j i}$. The conservative force is given by the equality

$$
F_{i j}^{C}\left(r_{i j}\right)= \begin{cases}a_{i j}\left(1-r_{i j} / r_{c}\right) & \text { for } r_{i j} \leq r_{c}, \\ 0 & \text { for } r_{i j}>r_{c},\end{cases}
$$

where $a_{i j}$ is the conservative force coefficient between particles $i$ and $j$, and $r_{c}$ is the cut-off radius.

Each of the forces is applied to the fluid particles as well as to the particles constituting $\mathrm{RBC}$ and platelet membranes.

If the following two relations are satisfied, the system will preserve its energy and maintain the equilibrium temperature:

$$
\omega^{D}\left(r_{i j}\right)=\left[\omega^{R}\left(r_{i j}\right)\right]^{2}, \quad \sigma^{2}=2 \gamma k_{B} T,
$$

where $k_{B}$ is the Boltzmann constant and $T$ is the temperature. The weight functions are determined by:

$$
\omega^{R}\left(r_{i j}\right)= \begin{cases}\left(1-r_{i j} / r_{c}\right)^{k} & \text { for } r_{i j} \leq r_{c}, \\ 0 & \text { for } r_{i j}>r_{c},\end{cases}
$$

where $k=1$ for the original DPD method, but it can be also varied in order to change the dynamic viscosity of the simulated fluid [12]. The motion of particles is determined by Newton's second law of motion:

$$
\Delta \mathbf{r}_{i}=\mathbf{v}_{i} \Delta t, \quad \Delta \mathbf{v}_{i}=\frac{\Delta t}{m_{i}} \sum_{j \neq i}\left(\mathbf{F}_{i j}^{C}+\mathbf{F}_{i j}^{D}+\mathbf{F}_{i j}^{R}\right),
$$

where $m_{i}$ is the mass of the particle $i$.

Euler method or a modified version of the velocity-Verlet method $[2,16]$, which is more accurate, can be used to integrate the equations (2.25). In the former,

$$
\begin{aligned}
\mathbf{v}_{i}^{n+1} & =\mathbf{v}_{i}^{n}+\frac{1}{m_{i}} \mathbf{F}_{i}\left(\mathbf{r}_{i}^{n}, \mathbf{v}_{i}^{n}\right) \Delta t, \\
\mathbf{r}_{i}^{n+1} & =\mathbf{r}_{i}^{n}+\mathbf{v}_{i}^{n+1} \Delta t,
\end{aligned}
$$


N. Bessonov, E. Babushkina, S.F. Golovashchenko A. Tosenberger, F. Ataullakhanov, M. Panteleev A. Tokarev, V. Volpert Numerical modelling of cell distribution in blood flow

where indices $n$ and $n+1$ denote time steps, and

$$
\mathbf{F}_{i}=\sum_{j \neq i}\left(\mathbf{F}_{i j}^{C}+\mathbf{F}_{i j}^{D}+\mathbf{F}_{i j}^{R}\right)
$$

The discretization in the second method is as follows:

$$
\begin{aligned}
\mathbf{r}_{i}^{n+1} & =\mathbf{r}_{i}^{n}+\mathbf{v}_{i}^{n} d t+\frac{1}{2} \mathbf{a}_{i}^{n} \Delta t^{2}, \\
\mathbf{v}_{i}^{n+\frac{1}{2}} & =\mathbf{v}_{i}^{n}+\frac{1}{2} \mathbf{a}_{i}^{n} \Delta t, \\
\mathbf{a}_{i}^{n+1} & =\frac{1}{m_{i}} \mathbf{F}_{i}\left(\mathbf{r}_{i}^{n+1}, \mathbf{v}_{i}^{n+\frac{1}{2}}\right), \\
\mathbf{v}_{i}^{n+1} & =\mathbf{v}_{i}^{n+\frac{1}{2}}+\frac{1}{2} \mathbf{a}_{i}^{n+1} \Delta t,
\end{aligned}
$$

where $a_{i}^{n}$ denotes the acceleration of the particle $i$ at the $n^{\text {th }}$ time step. Both methods give close results.

The following values of parameters were used: $r_{c}=1 \cdot 10^{-6} \mathrm{~m}, a_{i, j}=1 \cdot 10^{-12} \mathrm{~N}, \gamma=1 \cdot 10^{-8} \mathrm{~kg} \cdot \mathrm{sec}$ $/ \mathrm{m}^{4}, \sigma=1 \cdot 10^{-11} \mathrm{~kg} /\left(\mathrm{m}^{3} \cdot \sqrt{\mathrm{sec}}\right)$.

The behaviour of the DPD method, as well as its suitability for the problem of fluid simulation is well described in literature $[12,14,16,20,37]$. In $[12,14]$ DPD simulation results are compared with the results obtained by using continuous methods (Navier-Stokes and Stokes equations) for Couette, Poiseuille, square-cavity and triangular-cavity flow.

Let us note that in order to have a nonzero velocity field we should apply either nonzero flow velocity at the entrance of the domain or a volume force in the whole domain. These two approaches are basically equivalent though some difference can appear in non-cylindrical domains. Numerical simulations show that this difference is not essential for the results presented here. We used the approach with a volume force in our simulations.

The simulations were carried out in a cylindrical channel. The no-slip boundary condition at the cylinder wall and periodical boundary condition in the direction of the cylinder axis were used. Numerical realization of these boundary conditions is described in [39].

\section{Results}

We consider the following parameters of blood cells. We set for erythrocytes the equilibrium length of the edges $l_{0}=4.43 \cdot 10^{-7} \mathrm{~m}$, the triangle element area and the relaxation volume of erythrocytes $s_{0}=9.3 \cdot 10^{-14} \mathrm{~m}^{2}$ and $v_{0}=1.36 \cdot 10^{-16} \mathrm{~m}^{3}$ respectively. The mesh for each erythrocyte consists of 1280 triangles. For platelets, $l_{0}=6.18 \cdot 10^{-7} \mathrm{~m}, s_{0}=1.65 \cdot 10^{-13} \mathrm{~m}^{2}$ and $v_{0}=3.65 \cdot 10^{-18} \mathrm{~m}^{3}$. The mesh for each platelet consists of 80 triangles. Finally, we put for leucocytes $l_{0}=4.34 \cdot 10^{-7} \mathrm{~m}, s_{0}=1.6 \cdot 10^{-13}$ $\mathrm{m}^{2}$ and $v_{0}=2.27 \cdot 10^{-16} \mathrm{~m}^{3}$. The mesh for each leucocyte consists of 1280 triangles.

The mass of each particle for erythrocyte, platelet and leucocyte is $m=1 \cdot 10^{-19} \mathrm{~kg}$. The density of the membrane is approximately the same as the density of the surrounding fluid. Fluid particles are also inside cells with the same density as outside.

The relaxation volume of blood cells determine their properties. The value of $v_{0}$ for erythrocytes is taken about 0.5 of the volume of the corresponding sphere, whereas the value of $v_{0}$ for platelets equals the volume of the sphere. As a result, platelets remain almost spherical or ellipsoidal while erythrocytes take their specific biconcave shape. The leucocyte relaxation volume is chosen in the same way as for platelets.

In order to prevent cell penetration inside other cells, we use the soft contact algorithm [4].

In all simulations with the blood flow in this section we get an average flow velocity from $0.53 \mathrm{~mm} / \mathrm{s}$ to $1.6 \mathrm{~mm} / \mathrm{s}$. The effective viscosity is $4-5 \cdot 10^{-3} \mathrm{~Pa} \cdot \mathrm{s}$. The corresponding viscosity of the homogeneous fluid (without blood cells) is $1.4-2 \cdot 10^{-3} \mathrm{~Pa} \cdot \mathrm{s}$. These values correspond to experimental values of blood viscosity. Wall shear rate is about $2001 /$ sec. 
N. Bessonov, E. Babushkina, S.F. Golovashchenko A. Tosenberger, F. Ataullakhanov, M. Panteleev A. Tokarev, V. Volpert Numerical modelling of cell distribution in blood flow

\subsection{Stretching RBC}

One of the experimental methods to determine mechanical properties of erythrocytes is stretching by optical tweezers. The experiment provides the values of transversal and longitudinal diameters of a cell under two stretching loads [49]. In order to determine parameters of our erythrocyte model, we simulate this experiment numerically. We put a RBC with initial biconcave shape into a three-dimensional domain without flow. We choose several nodes from the opposite sides and apply a force to them. We stretch the cell by a force with the values used in the experiment and measure the diameters of a cell in two directions. The values of parameters for the models I, II and III are chosen to fit the experimental curves. This allows us also to compare different models. Figure 3 shows the scheme of the numerical experiment and comparison with experiments for the three models.
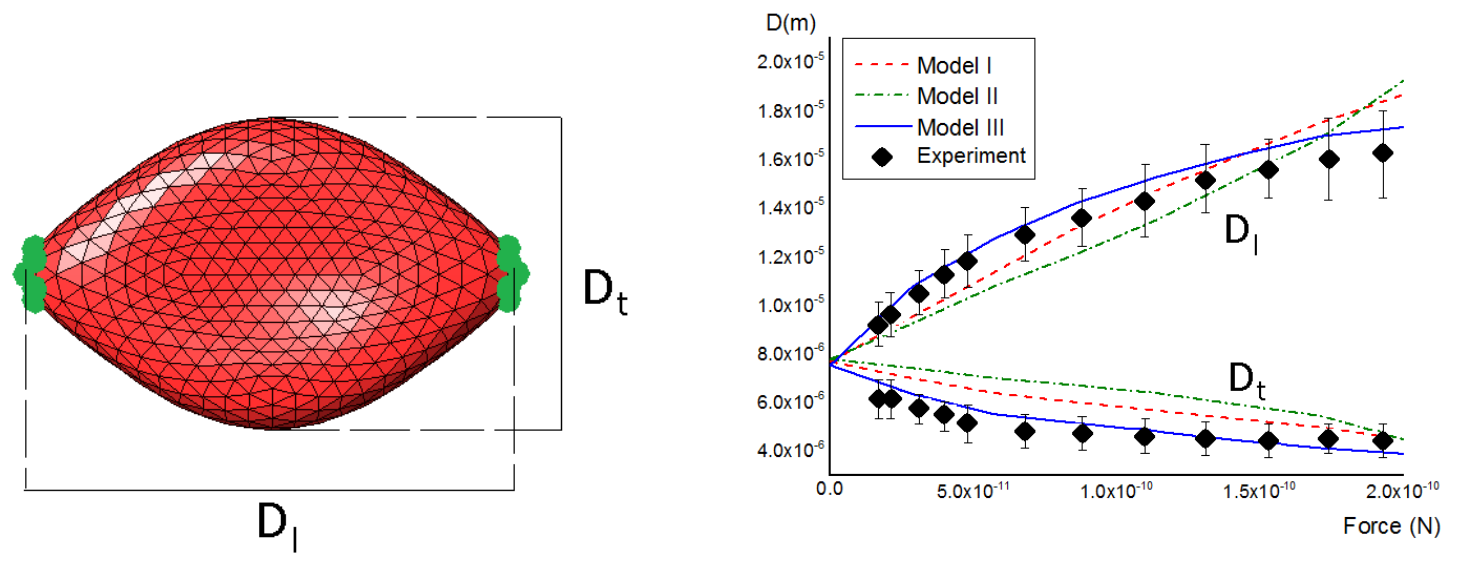

FiguRE 3. Stretching of erythrocyte (left), comparison of numerical simulations and experiments (right).

The following values of parameters are chosen to describe the experiment curves:

I: $k_{v}=124 \mathrm{~N} / \mathrm{m}^{2}, k_{b}=2.2 \cdot 10^{-11} \mathrm{~N}, k_{s}=6.45 \cdot 10^{-12} \mathrm{~N}, k_{a}=5 \cdot 10^{-5} \mathrm{~N} / \mathrm{m}$,

II: $k_{v}=124 \mathrm{~N} / \mathrm{m}^{2}, k_{b}=2.14 \cdot 10^{-11} \mathrm{~N}, K=1 \cdot 10^{-5} \mathrm{~Pa}, G=8.43 \cdot 10^{-6} \mathrm{~Pa}$,

III: $k_{v}=124 \mathrm{~N} / \mathrm{m}^{2}, k_{b}=1.62 \cdot 10^{-11} \mathrm{~N}, K=7.1 \cdot 10^{-6} \mathrm{~Pa}, G_{1}=4.85 \cdot 10^{-7} \mathrm{~Pa}, G_{2}=1.5 \cdot 10^{-6} \mathrm{~Pa}$.

It can be seen from Figure 3 that the Model III gives the best agreement between simulations and experimental results. We choose this model for all following simulations. For the other two models the dependence of the diameters on force is almost linear, and it differs from the experimentally observed one.

\subsection{Margination of erythrocytes and platelets}

Numerical simulations presented in this section were carried out for the radius $R$ of the cylindrical channel $13.5 \mu \mathrm{m}$. Its length was chosen in such a way that it did not influence the results. The value $H_{t}$ of hematocrit was taken 20,30 and $40 \%$ in different simulations. The value $40 \%$ corresponds to the normal hematocrit value in blood. The number of erythrocytes ranged from 45 to 90 depending on $H_{t}$.

The initial erythrocytes and platelets distribution is shown in Figure 4. The proportion of platelets was greater than in the normal blood. This accelerates their redistribution in flow and reduces the computational time. However, this does not practically influence their steady distribution.

The results of numerical simulations are shown in Figure 5 (a part of the computational domain). We obtain a steady cell distribution after 10-15 seconds of physical time. 

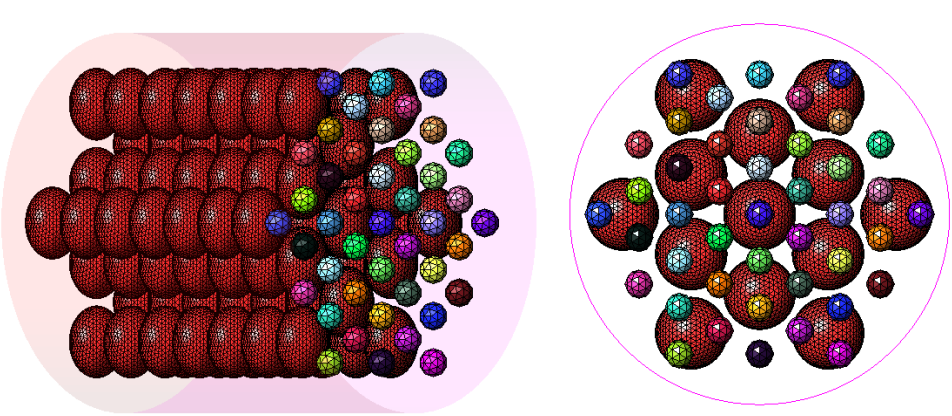

Figure 4. Cylindrical channel (left) and its cross-section (right) with initial erythrocyte and platelet distribution for $H_{t}=40 \%$
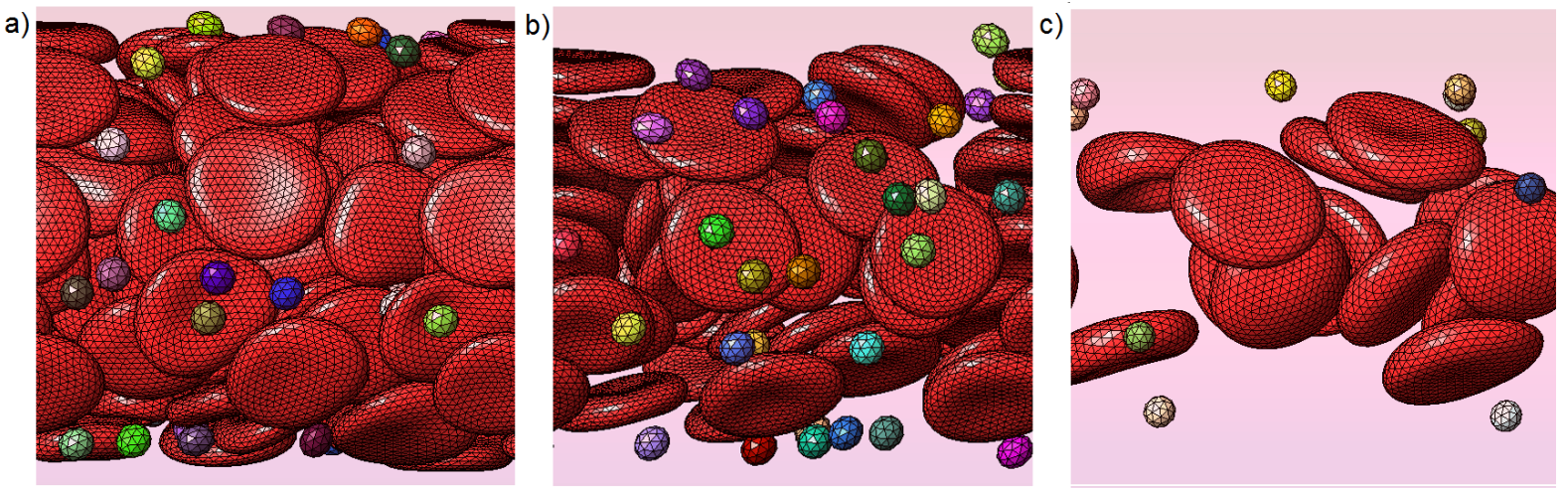

FiguRE 5. Snapshots of numerical simulations showing erythrocytes and platelets in the flow in cylindrical channel for three different values of $H_{t}$ : a) $40 \%$, b) $20 \%$, c) $10 \%$. Blood plasma particles, which occupy the remaining volume of the channel, are not shown.

In order to determine cell distribution in the cross section of the cylinder we divided it into n narrow annuli $S_{i}$ for which $r_{i}<r<r_{i+1}, r_{0}=0, r_{n}=R$. We counted the number $N_{i}$ of cell vertices belonging to each annulus during some sufficiently large time T. Next, we divide $N_{i}$ by the area of the corresponding annulus $S_{i}$. We call this ratio cell distribution in the cross section and denote it by $\mathrm{D}$. The distribution curves scaled by their maximum are shown in Figure 6 .

Figure 6 shows erythrocyte and platelet distributions in the cross section of cylinder for different values of $H_{t}$. Erythrocyte concentration gradually increases to the axis, while platelets are localized near the wall. This effect is well-known from the experiments $[21,33,34,42,43]$. A similar erythrocyte distribution is obtained in [1].

Figure 7 shows the average velocity along the radius of a cylinder. While the velocity profiles were parabolic in simulations with only plasma particles, in simulations with erythrocytes the velocity profiles were flat in the middle of the vessel because of the high erythrocyte concentration. The influence of cells presence became stronger with increasing of $H_{t}$ value. These results are in good agreement with the experimental data [15]. 

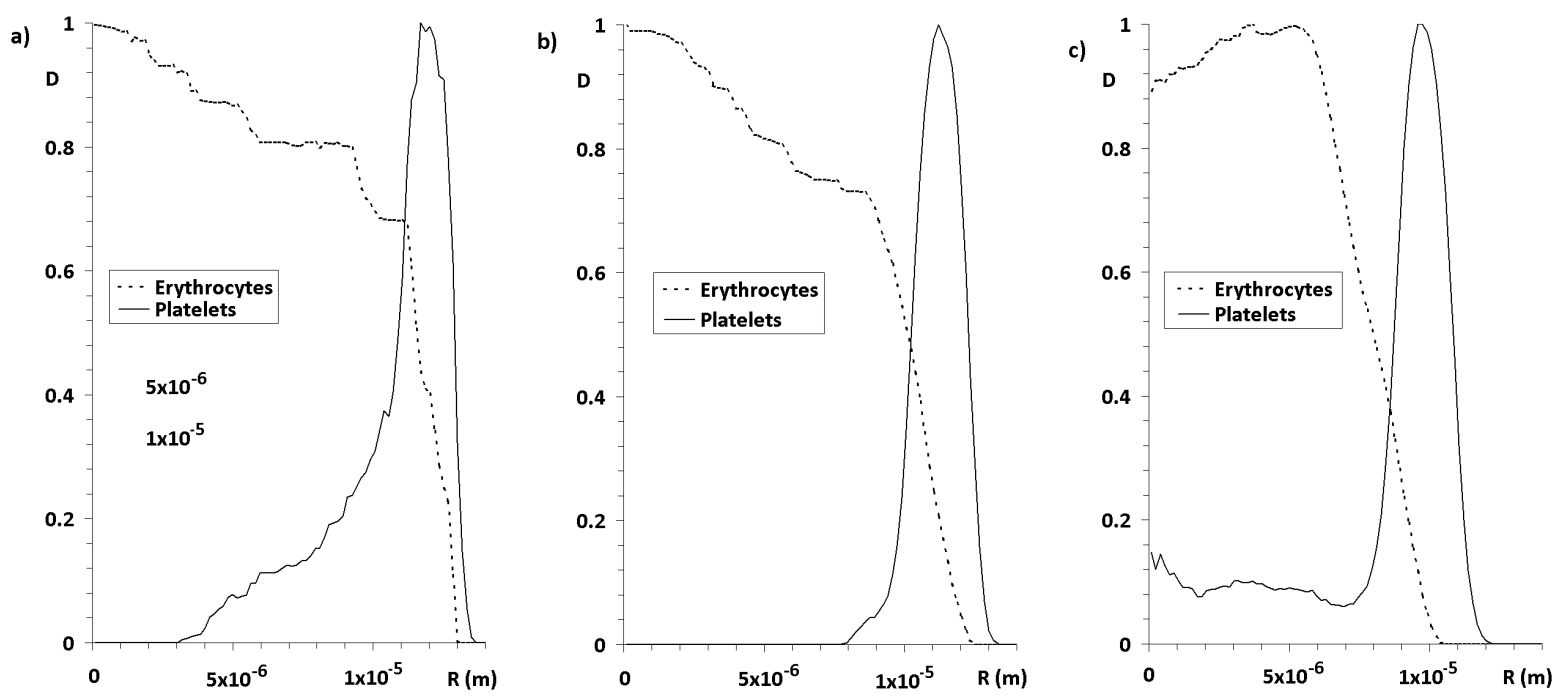

FIgURE 6. Erythrocyte (solid line) and platelet (dashed line) distributions as a function of distance from the axis for three values of $H_{t}$ : a) $40 \%$, b) $20 \%$, c) $10 \%$.

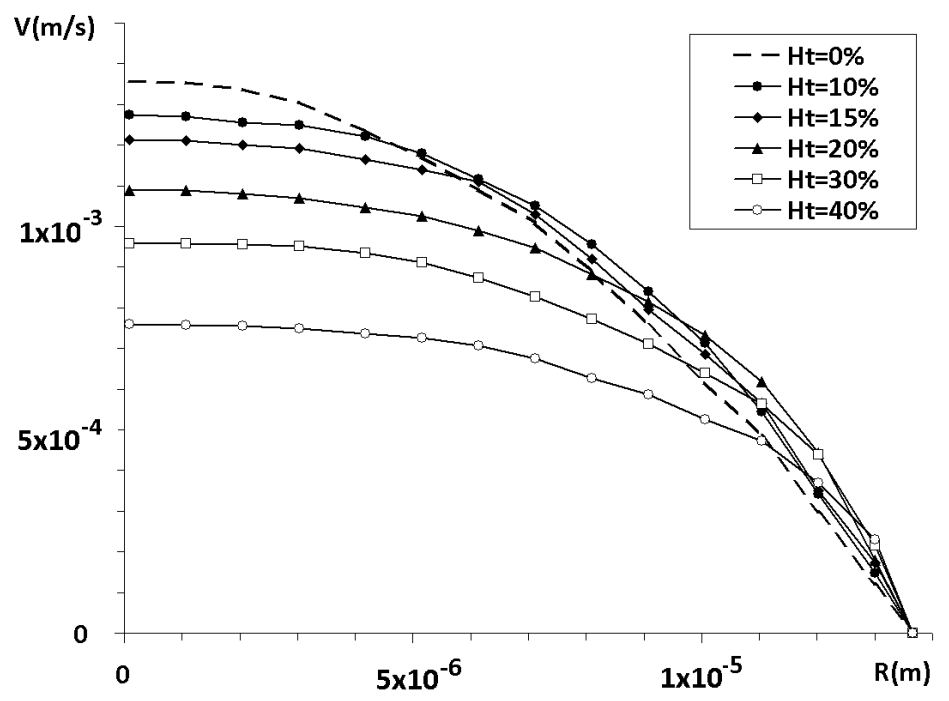

FiguRE 7. Averaged velocity profiles in simulations with erythrocytes and platelets. Velocity profiles are shown as functions of distance from the flow axis for the values of $H_{t}: 40 \%, 30 \%, 20 \%, 15 \%, 10 \%$ and without erythrocytes $\left(H_{t}=0\right)$.

\subsection{Vessels with variable cross section}

In order to model blood flow in a vessel with stenosis, we considered a cylinder with variable cross section where the largest radius was $20 \mu \mathrm{m}$ and the smallest one $10 \mu \mathrm{m}$ (Figure 8 (left)). The hematocrit level in the channel was $35 \%$. We can see from the figure that cells are densely packed before stenosis and there are cell free areas after it. Deformability of erythrocytes determines behavior of the flow when it passes 
the stenotic area. Figure 8 (right) shows the trajectories of randomly chosen erythrocyte and platelet. As it was for the cylinder with constant radius, platelets move along the vessel wall, whereas erythrocytes stay more close to the axis.

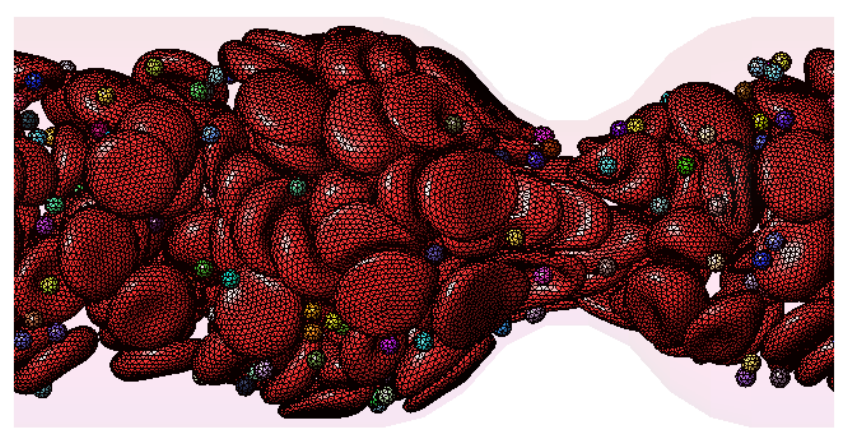

a)

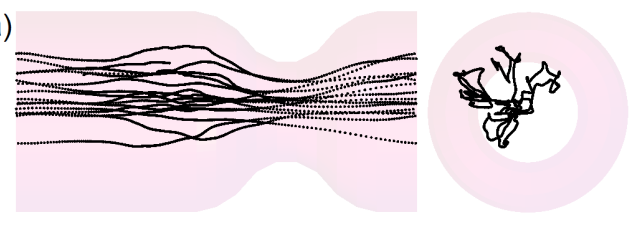

b)

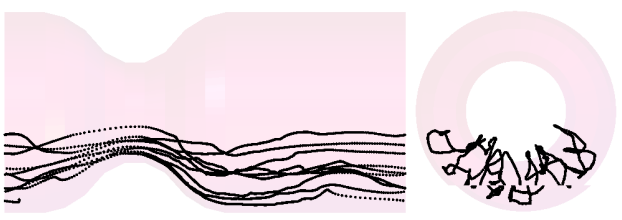

FiguRE 8. Left: blood flow in a channel with a narrower central part, right: a) trajectory of an erythrocyte, b) trajectory of a platelet.

We also studied the case where a part of the vessel was larger than the remaining part of the cylindrical channel (Figure 9). The radius of the channel was $13.5 \mu \mathrm{m}$ and the largest twice wider. As before, the hematocrit level was $35 \%$. Figure 9 (top) shows the results of the simulations. Important property of this flow is blood recirculation in the wider part of the vessel. It was not observed in the case of stenosis.

About $25 \%$ of platelets remain in the recirculation zone and about $4 \%$ of erythrocytes. Figure 9 (bottom) shows examples of cells trajectories in the flow. Three types of trajectories are observed for both cell types: cells are located in the wider part of the channel and do not leave it; cells move in the flow, but getting into the wider part of the vessel they remain there for some time; cells move in the flow and pass the recirculation zone. Let us note that blood recirculation occurs in varicosis, and it can provoke thrombosis. The model presented here can be used to study this process.

\subsection{Leukocyte behavior}

In this section we model behaviour of a leukocyte in blood flow with erythrocytes. The simulations was carried out for the cylinder with radius $R=22 \mu \mathrm{m}$. The value of $H_{t}$ was about $30 \%$. Since there are about $1 \%$ of leucocytes among all blood cells, we introduced one leukocyte and 100 erythrocytes (10 (left)).

It is well known from in vivo observations that WBC rolls along the vessel wall [23]. At the beginning of the simulations, the leucocyte was located at the axis of the cylinder. After some time, it was pushed by erythrocytes to the vessel wall. We can conclude that there is a stationary solution of this problem where the leucocyte is located at the axis but this solution is unstable.

Figure 10 (right) shows a snapshot of the numerical simulations, and Figure 11 (left) the dependence of the position of the leucocyte center on time. It moved to the vessel wall and continued moving along the wall. To check rolling of WBC we put a mark (green point) at its surface and traced its motion (Figure 11, right).

\section{Discussion}

In this paper we study three-dimensional hemodynamics with blood cells. Blood plasma is modelled with the DPD method. We have developed a new model for blood cells that combines particle description and 


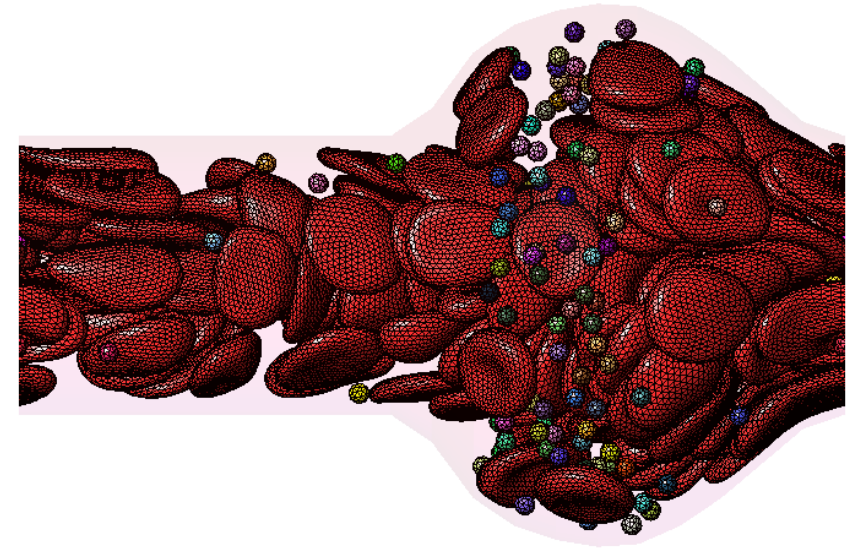

a)

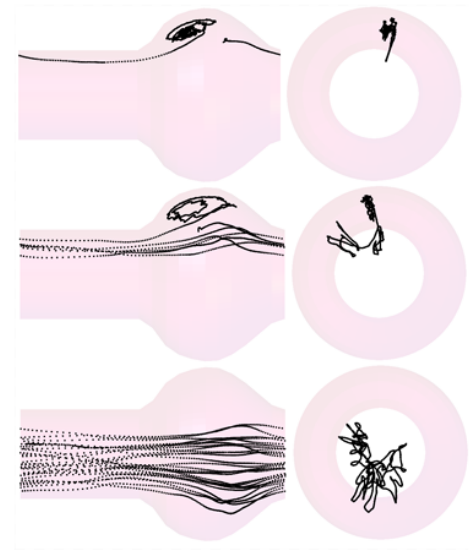

b)
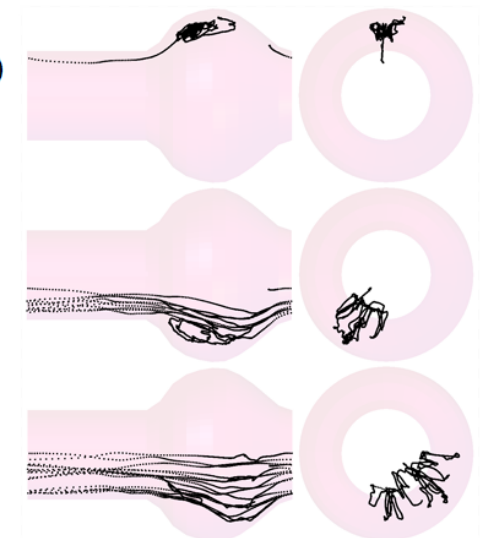

Figure 9. Top: blood flow in a channel with a wider central part, bottom: examples of a) erythrocyte b) platelets trajectories in the flow.

continuous elastic body model. This method allows us to obtain a good agreement between numerical simulations on erythrocyte stretching and experiments.

Using this new hybrid (discrete-continuous) method for blood cells and the DPD method for plasma, we obtain a realistic description of blood flow. We observe migration of erythrocytes to the axis, of platelets and white blood cells to the walls.

The method used in this work has some limitations. Blood cells have more complex structure and they can interact with each other forming aggregates. Furthermore, we do not take into account elastic properties of vessel walls. We can only consider microvessels because simulations are quite expensive from the computational point of view.

In spite of these limitations, the simulations described above can now be used to investigate various biomedical questions. For example, thrombosis and haemostasis are very important and challenging questions which need detailed modelling and simulations. This modelling can also be applied in diagnostics of various diseases, such as leukemia, that can change mechanical properties of blood cells. Another interesting question is simulation of blood flow in vessels with complex geometry. These questions will be studied in the forthcoming works.

Acknowledgements. The study was supported by the Russian Foundation for Basic Research grants 10-01-91055, 11-04-00303, 11-04-12080, 12-04-00652, 12-04-00438, 12-04-32095, 12-04-33055, 14-01-91055, Russian Federation 
N. Bessonov, E. Babushkina, S.F. Golovashchenko A. Tosenberger, F. Ataullakhanov, M. Panteleev A. Tokarev, V. Volpert Numerical modelling of cell distribution in blood flow

a) time $=0 \mathrm{sec}$

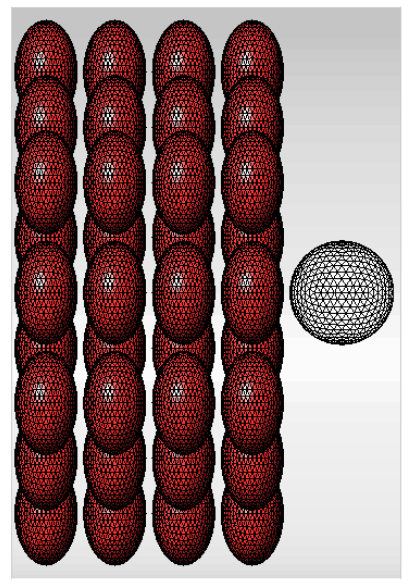

b) time $=30 \mathrm{sec}$

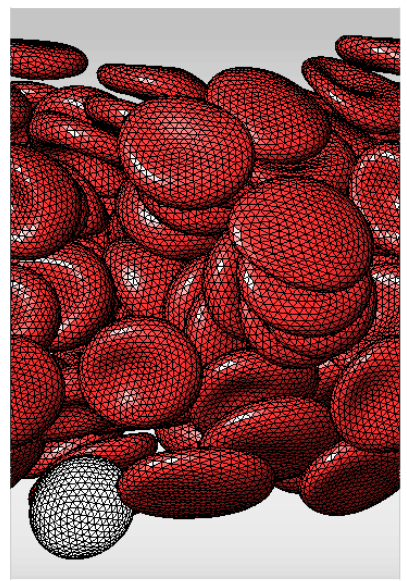

FiguRE 10. Initial cell distributions and their distribution after 30 seconds.
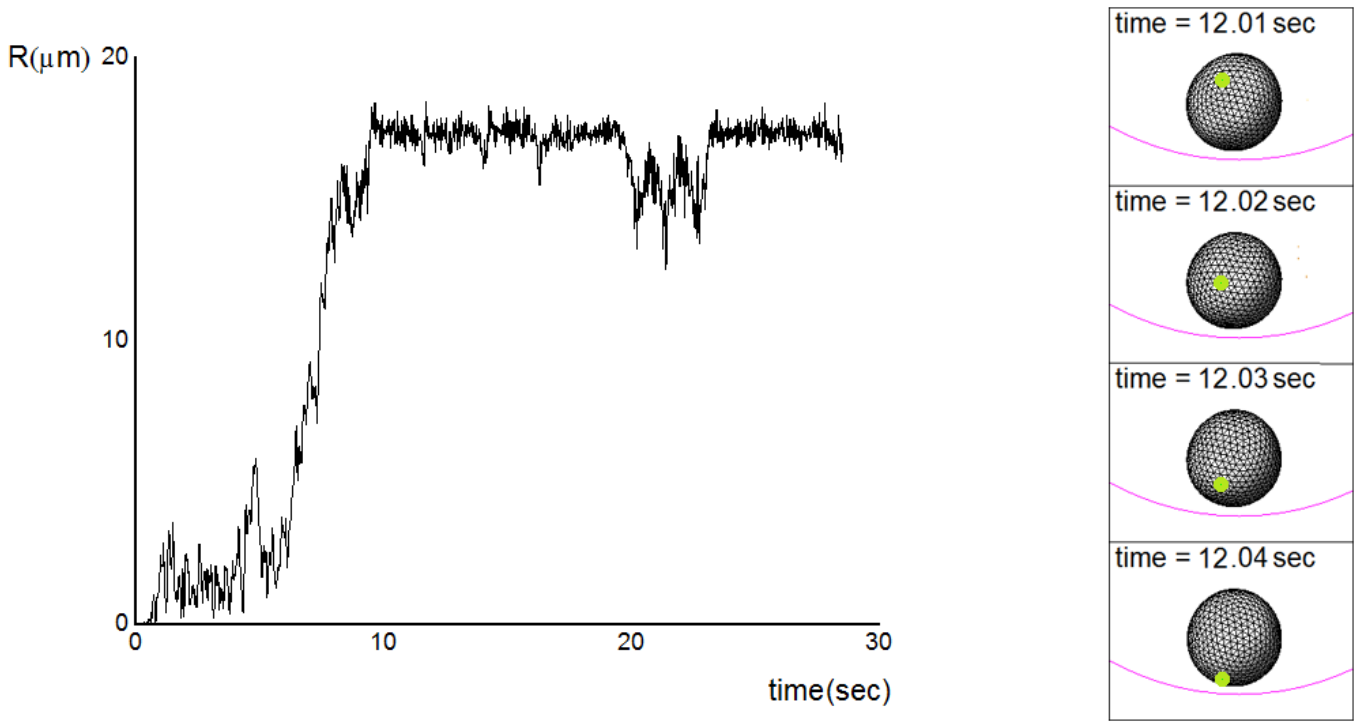

FiguRE 11. Left figure shows the distance of the leucocyte center to the vessel wall. Snapshots on the right demonstrate rolling of the leucocyte along the vessel wall.

Presidential Scholarship for Young Scientists and Graduate Students and by the Russian Academy of Sciences Presidium Basic Research Programs Molecular and Cellular Biology, Basic Science for Medicine, Integrative Physiology, and Molecular Mechanisms of Physiologic Functions.

N. Bessonov and V. Volpert were partially supported by the French ANR project Bimod, ANR-10-BLAN-010701 and by the grant no. 14.740.11.0877 of the Ministry of Education and Research of the Russian Federation, "Investigation of Spatial and Temporal Structures in Fluids with Applications to Mathematical Biology". 
N. Bessonov, E. Babushkina, S.F. Golovashchenko A. Tosenberger, F. Ataullakhanov, M. Panteleev A. Tokarev, V. Volpert Numerical modelling of cell distribution in blood flow

\section{References}

[1] D. Alizadehrad, Y. Imai, K. Nakaaki, T. Ishikawa, T. Yamaguchi. Parallel simulation of cellular flow in microvessels using a particle method. Journal of Biomechanical Science and Engineering, 7 (2012), no. 1, 57-71.

[2] M.P. Allen, D.J. Tidesley. Computer Simulation of Liquids. Clarendon, Oxford, 1987.

[3] T. AlMomani, H.S. Udaykumar, J.S. Marshall, K.B. Chandran. Micro-scale dynamic simulation of erythrocyte-platelet interaction in blood flow. Annals of Biomedical Engineering, Vol. 36 (2008), no. 6, 905-920.

[4] N. M. Bessonov, S.F. Golovashchenko, V.A. Volpert. Numerical Modelling of Contact Elastic-Plastic Flows. Math. Model. Nat. Phenom., Vol. 4 (2009), no. 1, 44-87.

[5] T. Bodnar, K. Rajagopal, A. Sequeira. Simulation of the Three-Dimensional Flow of Blood Using a Shear-Thinning Viscoelastic Fluid Model. Mathematical Modelling of Natural Phenomena, vol. 6 (2011), no. 5, 1-24.

[6] C. Bui, V. Lleras, O. Pantz. Dynamics of red blood cells in 2d. ESAIM: Proc., Vol. 28 (2009), 182-194.

[7] L.M. Crowl, A.L. Fogelson. Computational model of whole blood exhibiting lateral platelet motion induced by red blood cells. Int j numer method biomed eng., 26 (2010), no. 3-4, 471-487.

[8] M.M. Dupin, I. Halliday, C.M. Care, L. Alboul, L.L. Munn. Modeling the flow of dense suspensions of deformable particles in three dimensions. Physical Review E 75, 066707, 2007.

[9] W. Dzwinel, K. Boryczko, D.A. Yuen. Modeling Mesoscopic Fluids with Discrete-Particles Methods. Algorithms and Results., In: Spasic AM, Hsu JP (eds) Finely Dispersed Particles: Micro-, Nano-, and Atto-Engineering. Taylor \& Francis, CRC Press, 715-778.

[10] D. Fedosov, B. Caswell, G.E. Karniadakis. General coarse-grained red blood cell models: I. Mechanics. (2009), arXiv:0905.0042 [q-bio.CB].

[11] D. Fedosov, B. Caswell, G.E. Karniadakis. A Multiscale Red Blood Cell Model with Accurate Mechanics, Rheology, and Dynamics. Biophysical Journal, Volume 98 (2010), 2215-2225.

[12] D.A. Fedosov. Multiscale Modeling of Blood Flow and Soft Matter. PhD dissertation at Brown University, (2010).

[13] D.A. Fedosov, H. Lei, B. Caswell, S. Suresh, G.E. Karniadakis. Multiscale Modeling of Red Blood Cell Mechanics and Blood Flow in Malaria. PLoS Computational Biology, Vol. 7 (2011), no. 12, e1002270.

[14] D.A. Fedosov, I.V. Pivkin, G.E. Karniadakis. Velocity limit in DPD simulations of wall-bounded flows. J. Comp. Phys., 227 (2008), 2540-2559.

[15] H.L. Goldsmith, V.T. Turitto. Rheological aspects of thrombosis and haemostasis: basic principles and applications. Thrombosis and Haemostasis, 55 (1986), no. 3, 415-435.

[16] R.D. Groot, P.B. Warren. Dissipative particle dynamics: Bridging the Gap Between Atomistic and Mesoscopic Simulation. J. Chem. Phys., 107 (1997), no. 11, 4423-4435.

[17] S.M. Hosseini, J.J. Feng. A particle-based model for the transport of erythrocytes in capillaries. Chem. Eng. Sci. 64, (2009) 4488-4497.

[18] Y. Imai, H. Kondo, T. Ishikawa, C.T. Lim, T. Yamaguchi. Modeling of hemodynamics arising from malaria infection. Journal of Biomechanics, (2010), no. 43, 1386-1393.

[19] Y. Imai, K. Nakaaki, H. Kondo, T. Ishikawa, C.T. Lim, T. Yamaguchi. Margination of red blood cells infected by Plasmodium falciparum in a microvessel. Journal of Biomechanics, (2011), no. 44, 1553-1558.

[20] M. Karttunen, I. Vattulainen, A. Lukkarinen. A novel methods in soft matter simulations. Springer, Berlin, 2004.

[21] J.F. Koleski, E.C. Eckstein. Near wall concentration profiles of 1.0 and $2.5 \mu \mathrm{m}$ beads during flow of blood suspensions. Trans. Ann. Soc. Intern. Organs, 37 (1991), 9-12.

[22] P.W. Kuchel, E.D. Fackerell. Parametric-Equation Representation of Biconcave Erythrocytes. Bulletin of Mathematical Biology, 61 (1999), 209-220.

[23] M. B. Lawrence, T. A. Springer. Leukocytes roll on a selectin at physiological flow rates: distinction from and prerequisite for adhesion through integrins. Cell, 65 (1991), 859-873.

[24] R.C. Leif, J. Vinograd. The Distribution of Buoyant Density of Human Erythrocytes in Bovine Albumin Solutions. Proc Natl Acad Sci U S A., 51 (1964), no. 3, 520-528.

[25] S. Leibler, A.C. Maggs. Simulation of shape changes and adhesion phenomena in an elastic model of erythrocytes. Proc. Natl. Acad. Sci. USA, vol. 87 (1990), 6433-6435.

[26] L. Lopez, I.M. Duck, W.A. Hunt. On the shape of the erythrocyte. Biophys J., 8 (1968), no. 11, $1228-1235$.

[27] J.L. McWhirter, H. Noguchi, G. Gompper. Flow-induced clustering and alignment of vesicles and red blood cells in microcapillaries. PNAS, vol. 106 (2009), no. 15, 6039-6043.

[28] N. Mohandas, P.G. Gallagher. Red cell membrane: past, present, and future. Blood, 112 (2008), 3939-48.

[29] L.L. Munn, M.M. Dupin, Blood Cell Interactions and Segregation in Flow. Annals of Biomedical Engineering, Vol. 36 (2008), no. 4, 534-544.

[30] S. Muñoz San Martín, J.L. Sebastián, M. Sancho1, G. Álvarez. Modeling Human Erythrocyte Shape and Size Abnormalities, arXiv:q-bio/0507024 [q-bio.QM], 14 Jul 2005.

[31] H. Noguchi, G. Gompper. Shape transitions of fluid vesicles and red blood cells in capillary flows. PNAS, vol. 102 (2005), no. 40, 14159-14164.

[32] D. Obrist, B. Weber, A. Buck, P. Jenny. Red blood cell distribution in simplified capillary networks. Phil. Trans. R. Soc. A 2010 368, doi: 10.1098/rsta.2010.0045, 2010.

[33] D. Pinho, A. Pereira, R. Lima, T. Ishikawa, Y. Imai, T. Yamaguchi. Red blood cell dispersion in 100 $\mu$ m glass capillaries: the temperature effect. C.T. Lim and J.C.H. Goh (Eds.), WCB 2010, IFMBE Proceedings, 31 (2010), $1067-1070$. 
N. Bessonov, E. Babushkina, S.F. Golovashchenko A. Tosenberger, F. Ataullakhanov, M. Panteleev A. Tokarev, V. Volpert Numerical modelling of cell distribution in blood flow

[34] E. Pinto, B. Taboada, R. Rodrigues, V. Faustino, A. Pereira, R. Lima. Cell-free layer (CFL) analysis in a polydimethysiloxane (PDMS) microchannel: a global approach. WebmedCentral Biomedical Engineering, 4 (2013), no.8, WMC004374.

[35] I.V. Pivkin, G.E. Karniadakis. Accurate Coarse-Grained Modeling of Red Blood Cells. Physical review letters, PRL 101 (2008) 118105.

[36] C. Pozrikidis. Modeling and Simulation of Capsules and Biological Cells. by Chapman \& Hall/CRC, ISBN (2003) 1-58488-359-6.

[37] U.D. Schiller. Dissipative Particle Dynamics. A Study of the Methodological Background. Diploma thesis at Faculty of Physics University of Bielefeld, 2005.

[38] A.A. Tokarev, A.A. Butylin, E.A. Ermakova, E.E. Shnol, G.P. Panasenko, F.I. Ataullakhanov. Finite Platelet Size Could Be Responsible for Platelet Margination Effect. Biophysical Journal, Vol. 101 (2011), 1835-1843.

[39] A. Tosenberger, V. Salnikov, N. Bessonov, E. Babushkina, V. Volpert. Particle Dynamics Methods of Blood Flow Simulations. Math. Model. Nat. Phenom., 6 (2011), no. 5, 320-332.

[40] K. Tsubota, S. Wada. Elastic force of red blood cell membrane during tank-treading motion: Consideration of the membraneâ's natural state. International Journal of Mechanical Sciences, 52 (2010), 356-364.

[41] K. Tsubota, S. Wada, H. Kamada, Y. Kitagawa, R. Lima, T. Yamaguchi. A Particle Method for Blood Flow Simulation, Application to Flowing Red Blood Cells and Platelets. Journal of the Earth Simulator, vol. 5 (2006), 2-7.

[42] C. Yeh, A.C. Calvez, E.c. Eckstein. An estimated shape function for drift in a platelet-transport model. Biophysical journal, vol. 67 (1994), 1252-1259.

[43] C. Yeh, E.c. Eckstein. Transient Lateral Transport of Platelet-Sized Particles in Flowing Blood Suspensions. Biophysical journal, vol. 66 (1994), 1706-1716.

[44] J. Zhang, P.C. Johnson, A.S. Popel. Effects of Erythrocyte Deformability and Aggregation on the Cell Free Layer and Apparent Viscosity of Microscopic Blood Flows. Microvasc Res., 77 (2009), no. 3, 265-272.

[45] P. Bagchi. Mesoscale simulation of blood flow in small vessels. Biophysical Journal, 92 (2007), no. 6, 1858-1877 [PubMed: 17208982].

[46] R. Skalak, A. Tozeren, R. Zarda, S. Chein. Strain energy function of red blood cell membranes. Biophysical Journal 13 (1973), no. 3, 245-264 [PubMed: 4697236].

[47] A.A. Tokarev, A.A. Butylin, F.I. Ataullakhanov. Platelet Adhesion from Shear Blood Flow Is Controlled by Near-Wall Rebounding Collisions with Erythrocytes. Biophys. J., 100 (2011), no. 4, 799-808.

[48] A.A. Tokarev, A.A. Butylin, F.I. Ataullakhanov. Platelet transport and adhesion in shear blood flow: the role of erythrocytes. Computer Research and Modeling, 4 (2012), no. 1, 185-200 [article in Russian].

[49] S. Suresh, J. Spatz, J. P. Mills, A. Micoulet, M. Dao, C. T. Lim, M. Beil, T. Seufferlein. Connections between single-cell biomechanics and human disease states: gastrointestinal cancer and malaria. Acta Biomaterialia, 1 (2005), 15-30. 